\title{
SpineCor vs rigid brace for Adolescent Idiopathic Scoliosis: end of growth results from a retrospective controlled study
}

\author{
Fabio Zaina*, Fabio Digiacomo, Sabrina Donzelli, Michele Romano, Alessandra Negrini, Monia Lusini, \\ Salvatore Minnella, Stefano Negrini \\ From 10th International Conference on Conservative Management of Spinal Deformities - SOSORT 2013 \\ Annual Meeting \\ Chicago, IL, USA. 8-11 May 2013
}

\section{Background}

SpineCor and rigid braces both have both results testifying to their effectiveness in Adolescent Idiopathic Scoliosis (AIS) treatment: an RCT recently compared the two, showing the superiority of rigid braces. In a previous study, we found similar short- term results in curves between $20^{\circ}$ and $30^{\circ} \mathrm{Cobb}$.

\section{Purpose}

The objective of this study was to compare the shortterm results of the Spinecor vs SPoRT brace for AIS in this selected population.

\section{Methods}

Study design: retrospective controlled study. Population: Rigid Brace Groups (RBGs) 20 patients (16 female), age $13 \pm 1$, Cobb $24 \pm 5^{\circ}$, ATR $8 \pm 3^{\circ}$, TRACE score 7 , Risser 0-3. Spinecor Group (SG): 41 patients (33 females) age $13 \pm 1$, Cobb angle $24 \pm 5^{\circ}$, ATR $8 \pm 3^{\circ}$, TRACE score 6 , Risser 0-3. Both groups were treated with a full-time brace (18 to 23 hours per day upon initial prescription). Clinical and radiological evaluations were performed at the beginning and end of treatment. Main outcome measures: Cobb angle (changes $> \pm 5$ ), ATR, TRACE (changes $\geq 3$ ). Statistics: Chi square, t-test.

\section{Results}

Considering patients with more than $5^{\circ}$ of Cobb angle change, $40 \%$ improved, $45 \%$ remained stable and $15 \%$ worsened in the RBG vs. $22 \%, 34 \%$ and $44 \%(p<0.05)$, respectively. No differences were found for ATR. For TRACE, there were no differences among groups: in RBG, $50 \%$ improved and $50 \%$ were stable, vs. $65 \%$, $31 \%$ and $5 \%$ worsened $(\mathrm{p}>0.05)$.

\section{Conclusions and discussion}

Both treatments showed to be effective in improving the aesthetics in AIS. For the other parameters, the SPoRT brace seemed to be more effective than the SpineCor to avoid curve progression, since the number of worsened patients was much higher for the SG. The main limits of the study were the retrospective design and the small population, so further studies are required.

Published: 18 September 2013

\section{References}

1. Zaina F, Donzelli S, Negrini A, Romano M, Negrini S: SpineCor, exercise and SPoRT rigid brace: what is the best for Adolescent Idiopathic Scoliosis? Short term results from 2 retrospective studies. Stud Health Technol Inform 2012, 176:361-4.

2. Wong MS, Cheng JC, Lam TP, Ng BK, Sin SW, Lee-Shum SL, Chow DH, Tam SY: The effect of rigid versus flexible spinal orthosis on the clinical efficacy and acceptance of the patients with adolescent idiopathic scoliosis. Spine (Phila Pa 1976) 2008, 33(12):1360-5.

3. Negrini S, Aulisa AG, Aulisa L, Circo AB, de Mauroy JC, Durmala J, et al: 2011 SOSORT guidelines: Orthopaedic and Rehabilitation treatment of idiopathic scoliosis during growth. Scoliosis 2012, 7(1):3.

doi:10.1186/1748-7161-8-S2-O56

Cite this article as: Zaina et al: SpineCor vs rigid brace for Adolescent Idiopathic Scoliosis: end of growth results from a retrospective controlled study. Scoliosis 2013 8(Suppl 2):O56.

* Correspondence: fabio.zaina@isico.it

ISICO Italian Scientific Spine Institute, Milan, Italy

(c) 2013 Zaina et al; licensee BioMed Central Ltd. This is an Open Access article distributed under the terms of the Creative Commons Attribution License (http://creativecommons.org/licenses/by/2.0), which permits unrestricted use, distribution, and reproduction in any medium, provided the original work is properly cited. 